\title{
Ureteral mass in a patient with localized prostate adenocarcinoma a rare metastatic location
}

\begin{abstract}
A 69-aged man with localized adenocarcinoma prostate cancer, treated with radical radiotherapy and intermittent hormonal therapy 12 years ago, initiate with objective hematuria. CT scan showed a retroperitoneal mass infiltrating all along the right ureter with hydronephrosis. No other metastatic involvement was observed but PSA raised until 120ng/ $\mathrm{dl}$ (previous normal). As ureteral metastases from a prostate adenocarcinoma are extremely rare, we made a differential diagnosis between the more common entities affecting the upper urinary tract and retroperitoneum. Finally, the biopsy of the mass revealed infiltration from prostate cancer. Thus, we present a patient with really atypical dissemination of prostate adenocarcinoma with parallel rising PSA-levels but without any other metastatic disease.
\end{abstract}

Volume 8 Issue I - 2017

\author{
Elizabeth Jiménez,' Daniel Castellano,' María \\ Garrido, ${ }^{2}$ Guillermo de Velasco' \\ 'Department of Medical Oncology, University Hospital 12 de \\ Octubre, Spain \\ ${ }^{2}$ Department of Medical Oncology, University Hospital Severo \\ Ochoa, Spain
}

Correspondence: Elizabeth Jim?nez Aguilar, MD, University Hospital, Madrid, Avda de Cordoba, Spain, Email roy.sebastian@usat.edu

Received: May 09, 2017 | Published: May 12, 2017

\section{Clinical presentation}

A former smoker 69 -aged man graphic arts worker, with a past medical history of hypertension, hemicolectomy due to colon cancer 30 years ago (we ignore the disease state because no informs were provided), was diagnosed in 2004 of intermediate localized adenocarcinoma prostate cancer, Gleason $8(4+4)$ (T3N0M0). He received 6 months of neoadjuvant hormonotherapy (LH-RH analogues) and radical radiotherapy (76Gy) including prostate and seminal vesicles. PSA nadir was $0.27 \mathrm{ng} / \mathrm{ml}$, and patient continued hormonal therapy as adjuvant treatment for 2 years. In June 2016, patient presented with hematuria and CT scan showed a retroperitoneal mass infiltrating all along the right ureter with secondary grade 2 hydronephrosis (Figure 1). Routine laboratory analysis was normal. Bone scan had no pathological alteration.

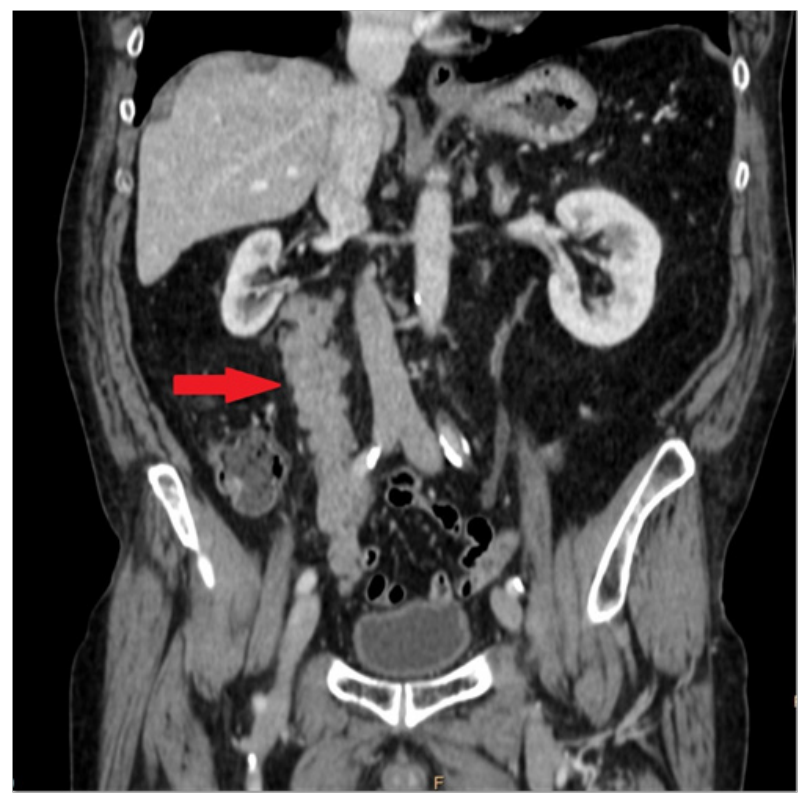

Figure I CT scan showed a retroperitoneal mass infiltrating all along the right ureter with secondary grade 2 hydronephrosis.

\section{What is your diagnosis?}

A. Urothelial carcinoma of the upper urinary tract

B. Retroperitoneal fibrosis

C. Metastatic adenocarcinoma prostate cancer

D. Primary Ureteral lymphoma

\section{Diagnosis}

Metastatic prostate cancer

\section{Discussion}

Tumors of the upper urinary tract are estimated to represent about $10 \%$ of all renal tumors. ${ }^{1}$ They are more frequently observed on renal pelvis than ureter. ${ }^{2}$ Urothelial carcinoma of the upper urinary tract is the most frequent malignant tumor arising from the renal pelvis $(90 \%)$. Other include squamous cell carcinomas $(10 \%){ }^{3}$ primary adenocarcinomas, and occasionally others tumor may be diagnosed such as small cell carcinomas, primary lymphoma, micropapillary or sarcomatoid patterns. ${ }^{4}$ Hematuria (presented in this patient) and/or ureterohydronephrosis obstruction are the most common symptoms at diagnosis. Urothelial carcinoma diagnosis is based on image and/ or urine cytology plus biopsy. A ureteral mass is typically found involving retrograde obstruction. The treatment for localized stage is nephroureterectomy. ${ }^{5}$ Interestingly, in this patient the mass was not causing an obstruction in the ureter, being less likely the diagnosis of urothelial cancer.

Although unusual, retroperitoneal fibrosis is a well-documented late side effect after radiotherapy. Presence of a fibro-inflammatory tissue extending into the retroperitoneum may involve ureters, usually leading to ureter obstruction. ${ }^{6}$ Retroperitoneal fibrosis is generally idiopathic occurring in men of 40-60 year old. Also, it has been reported secondary to multiple causes such as drugs, tumors or surgery or as long-term complication of radiation treatment for prostate cancer. Ureteral lymphoma is particularly rare because there is not lymphoid tissue in the ureteral wall. ${ }^{7}$ The PET/CT is the preferred study for the evaluation of the best site to perform the biopsy (fine 
needle aspiration is inadequate). Diagnosis depends on morphology, immunohistochemistry and flow cytometry. ${ }^{8}$

Our patient had a retroperitoneal mass infiltrating the right ureter with secondary grade 2 hydronephrosis. No other lymph nodes or metastases were observed. Biopsy of the mass revealed infiltration from prostate adenocarcinoma. Ureteral metastases from a prostate adenocarcinoma are extremely rare. ${ }^{9}$ The most common malignant tumors metastasizing to the ureter are breast followed by stomach and colorectal cancer. The incidence of prostatic originated metastasis is very low, with only 43 cases reported in the last century. ${ }^{9}$ Implantation could be produced by instrumentation, arterial embolism, and retrograde venous or lymphatic dissemination. The last one is the most accepted theory, but it would not match in this case because lymph node involvement was not present. ${ }^{10}$ Unlike most reported cases, our patient did not present any other metastatic disease. In parallel PSA value raised to $120 \mathrm{ng} / \mathrm{ml}$, with $35 \mathrm{ng} / \mathrm{dl}$ of testosterone, and hematuria stopped spontaneously.

As a result, we present a patient with really atypical dissemination of prostate adenocarcinoma located on the right ureter, confirmed by biopsy, with parallel rising PSA-levels but without any other metastatic disease.

\section{Funding}

None.

\section{Acknowledgements}

We want to acknowledge to the patient and his family for the permission of using these data.

\section{Conflicts of interests}

The authors declare that there is no conflict of interest.

\section{References}

1. Siegel R L, Miller K D, Jemal A. Cancer statistics, 2016. CA Cancer J Clin. 2016;66(1):7-30.

2. Oosterlinck W, Solsona E, Van Der Meijden APM, et al. EAU guidelines on diagnosis and treatment of upper urinary tract transitional cell carcinoma. Eur Urol. 2004;46(2):147-154.

3. Li MK, Cheung W L. Squamous cell carcinoma of the renal pelvis. $J$ Urol. 1987;138(2):269-271.

4. Holmäng S, Lele SM, Johansson SL. Squamous cell carcinoma of the renal pelvis and ureter: incidence, symptoms, treatment and outcome. $J$ Urol. 2007;178(1):51-56.

5. Lughezzani G, Sun M, Perrotte P, et al. Should bladder cuff excision remain the standard of care at nephroureterectomy in patients with urothelial carcinoma of the renal pelvis? A population-based study. Eur Urol. 2010;57(6):956-962.

6. Vaglio A, Salvarani C, Buzio C. Retroperitoneal fibrosis. Lancet Lond Engl. 2006;367(9506):241-251.

7. Schniederjan SD, Osunkoya AO. Lymphoid neoplasms of the urinary tract and male genital organs: a clinicopathological study of 40 cases. Mod Pathol. 2009;22(8):1057-1065.

8. Cheson BD, Fisher RI, Barrington S F, et al. Recommendations for initial evaluation, staging, and response assessment of Hodgkin and non-Hodgkin lymphoma: the Lugano classification. J Clin Oncol. 2014;32(27):3059-3068.

9. Schneider S, Popp D, Denzinger S, et al. A rare location of metastasis from prostate cancer: hydronephrosis associated with ureteral metastasis. Adv Urol. 2012:656023.

10. Singh G, Tiong H Y, Kalbit T, et al. Urothelial metastasis in prostate adenocarcinoma. Ann Acad Med Singapore. 2009;38(2): 170-171. 